\title{
Statistical vs. Identified Lives in Benefit-Cost Analysis
}

\author{
James K. Hammitt ${ }^{\mathrm{a}}$ and Nicolas Treich ${ }^{\mathrm{b}^{*}}$
}

May 2007

\footnotetext{
${ }^{a}$ Center for Risk Analysis, Harvard University 718 Huntington Ave., Boston, MA 02115 USA tel: 1617432 4343; e-mail: jkh@harvard.edu

${ }^{\mathrm{b}}$ Toulouse School of Economics (LERNA-INRA) Aile Jean-Jacques Laffont 21, allée de Brienne, Manufacture des Tabacs 31000 Toulouse, France tel: +33 (0)5 611285 14; e-mail: ntreich@toulouse.inra.fr

* This work began while JKH was appointed to a Pierre-de-Fermat Chaire d'Excellence at the Université de Toulouse. The authors thank Matthew Adler, Joanne Linerooth-Bayer, John Graham, Kip Viscusi, an anonymous referee, and seminar participants at the universities of Montréal, Paris (Panthéon-Sorbonne), Ohio State, Kyoto, Lille, Mannheim, and Harvard for helpful comments. JKH thanks the Université de Toulouse (LERNA-INRA, IDEI), ICSI, and the Région Midi-Pyrénées for hospitality and support.
} 


\begin{abstract}
Evaluation of projects that affect mortality risk usually assumes that risk changes are small and similar across individuals. In reality, risks differ among individuals and information about risk heterogeneity determines the extent to which affected lives are "statistical" or "identified" and influences the outcome of benefit-cost analysis (BCA). The effects of information about risk heterogeneity on BCA depend on, inter alia, whether information concerns heterogeneity of baseline or change in risk and whether valuation uses compensating or equivalent variation. BCA does not systematically favor identified over statistical lives. We suggest some political factors that may explain the apparent public bias.
\end{abstract}

Keywords: benefit-cost analysis, value of statistical life, information, heterogeneity JEL: D61, D81, H42, I18 


\section{Introduction}

Modern societies seem to spend more generously to save the lives of identified than anonymous victims (Viscusi, 2000a; Lamm, 2001; Richardson and McKie, 2003, Hsu, 2007), a phenomenon that has been described as "statistical murder." ${ }^{1}$ Spending large amounts to benefit only a few may be inefficient. It may also be inequitable: the question arises why some receive particular attention and not others. A bias toward identified lives can drive priorities in risk management including the choice between prevention and treatment of injury or disease (Corso et al., 2002) and the allocation of efforts among risks, e.g., treating kidney-disease patients or preventing traffic crashes, building nuclear or coal-fired power plants. From a broader perspective, information about whom a policy affects may influence the chance that it will be adopted. This effect may be particularly important for health and safety policies and for the valuation of health and mortality risk.

The economic literature on the "value of life" has from the beginning distinguished between identified and statistical lives. In the first paragraph of his seminal paper, Schelling (1968) writes “It is not the worth of human life that I shall discuss, but of 'life-saving,' of preventing death. And it is not a particular death, but a statistical death." Economists have since been cautious to make clear that the standard valuation approach - purposely labeled the "value of statistical life" (VSL) approach - applies only when changes in risk are small and similar among the affected population. ${ }^{2}$ As Viscusi (1992: 17) states, “This need to think in terms of statistical lives as opposed to certain lives defines the main character of our choice problem." The focus on statistical rather than identified lives is consistent with the traditional marginal approach to the valuation of public goods (Samuelson, 1954; Bergstrom, 1982) and lessens the scope for psychological concerns in the usual case where the effects of a policy on individual mortality risks are small (Schelling, 1968).

${ }^{1}$ In her paper “Murdering Statistical Lives ...?” Linnerooth (1982: 231) attributes the provocative term to Howard Raiffa. It was subsequently used by Graham (1995) and Viscusi (2000b). Tal (1998: 170) cites an alternative use by Merrell and Van Strum (1990: 21) to describe the permitting of any activity (e.g., pesticide use) that imposes mortality risk on someone. ${ }^{2}$ Howard (1984) proposed valuing risk increments in terms of "micromorts," i.e., risk changes of one in a million. This alternative term emphasizes the focus on small risk changes. Equivalent terms include value of statistical life (Viscusi, 1992), value per statistical life (Hammitt, 2000), value per life saved (Jones-Lee, 1976), and value of prevented fatality (Jones-Lee, 2004). 
The focus on statistical lives leaves open the question of how to evaluate a project that may save, or threaten, the lives of identified people. One may judge that the VSL approach is inappropriate when changes in individual risks are large. But what should one do instead? Scholars have recognized that identified and statistical lives are not the same (Schelling, 1968; Mishan, 1971; Weinstein et al., 1980; Keeney, 1995; Pratt and Zeckhauser, 1996) ${ }^{3}$ but how this difference should affect societal decisions is less clear.

Societies may choose to protect individual over statistical lives to support principles of respect for individuals and compassion (Zeckhauser, 1975; Tal, 1998). Decisions that are seen to protect identified victims have the merit of endorsing social values of compassion, solidarity, and unwillingness to sacrifice the few for the benefit of the many. In making this choice, societies must determine when the gains in equity, symbolism, and other objectives outweigh the loss in efficiency (Okun, 1975; Linnerooth, 1982). Differences between ex ante and ex post identifiability may have implications for decision making. Ex ante identifiability may attract public attention and invoke a "rule of rescue” response (Richardson and McKie, 2003). Ex post identifiability may be required to attribute deaths to particular decisions and to hold decision makers accountable (Raiffa et al., 1977).

The distinction between identified and statistical lives is not sharp but a continuum characterized by available information about heterogeneity of risk. People are neither identical nor anonymous and neither baseline risks nor policy-induced changes in risk are always small or identical across people. In general, baseline risks and risk changes differ among individuals because of differences in exposure (e.g., to sources of pollution, traffic conditions) and differences in susceptibility (e.g., related to age, sex, health status, genetics, behavior). In practice, information about how risks and policy-induced changes in risk differ among individuals varies among cases. Often information is so limited that one has little choice but to treat the risks as identical. Individuals may be conceived as facing a compound lottery. In the first stage, their baseline risk and policy-induced risk change are drawn from the population distribution and in the second stage the outcome of the mortality risk is realized. Before information about the outcome of the first stage is available, risks are identical across individuals

${ }^{3}$ In particular, Pratt and Zeckhauser (1996) examine the effects of risk and wealth on optimal public safety expenditures defined behind a Rawlsian veil of ignorance (before individuals have information about their own wealth and risk). Linnerooth (1982) and Hammitt (2002) compare VSL with effectiveness measures such as lives and life years saved. 
and the lives at risk are "statistical." If the outcome of the first stage is known and heterogeneity of risk is large, then the lives at risk are "identified.” Hence the identifiability of individuals at risk may be characterized by the quality of information about the heterogeneity of risk in the population.

The objective of this paper is to examine how information about heterogeneity of baseline risk and of policy-induced changes in risk influences economic valuation. This objective can be efficiently studied using the standard one-period model. Specifically, we investigate how the results of benefit-cost analysis (BCA) depend on information about heterogeneity of individual risks and risk changes in the conventional static VSL model. We compare these results with maximization of social welfare, defined as the mean of individuals' expected utilities (Harsanyi, 1953, 1955). This measure of social welfare is independent of information about risk heterogeneity when, as in our analysis, people are otherwise identical. ${ }^{4}$

We show that information about risk heterogeneity has, in many cases, a predictable effect on the outcome of BCA. In the usual case in which one aggregates individuals' willingness to pay for a project that reduces risk, information about heterogeneity of risk change decreases the value of the project. This result contrasts with the "identifiability bias" or the "identifiable-victim effect" that has been discussed in the literature in psychological and behavioral law and policy (e.g., Small and Lowenstein, 2003; Hsu, 2007).

Our analysis confronts a well-known conceptual issue, "Broome’s paradox."들 Consider a project that is anticipated to kill one person from a population of similar individuals (e.g., the project may put construction workers at risk). It may be that if the identity of the one who would die were unknown the project would pass a net-benefit test and be adopted. Yet if this person's identity were known he might require infinite compensation for the loss of his life and the project would fail the net-benefit test and be rejected. This is a paradox in the sense that the only difference between the two situations is knowing the identity of the person who will die, and this

\footnotetext{
${ }^{4}$ Another critical source of heterogeneity is wealth. The effects of wealth heterogeneity are beyond the scope of this paper. We concentrate on the issue announced in the title, which motivates the focus on heterogeneity of risk.

${ }^{5}$ Broome's (1978) provocative paper raises a number of issues for BCA. Many of these issues are addressed in a set of critical papers (Buchanan and Faith, 1979; Jones-Lee, 1979; Williams, 1979). On the issue of interest to this paper, Ulph (1982) suggests that Broome's paradox arises due to an inappropriate social welfare function, i.e., a utilitarian framework. Our discussion differs from Ulph’s since we work within a utilitarian framework.
} 
it may be argued (Broome, 1978) should not ultimately affect the social decision rule. ${ }^{6}$ Broome's paradox is a useful starting point for our analysis.

The remainder of the paper is organized as follows. In Section 2, we present a simple example based on Broome's paradox that highlights the effect of information about heterogeneity of policy-induced changes in mortality risk on BCA. In Section 3, we review the standard model of value per statistical life. Using that model, we examine in Section 4 how information about the heterogeneity of policy-induced changes in mortality risk affects each of the four measures of economic valuation: compensating and equivalent variations for reductions and increases in mortality risk. We find that the effect of more information about the heterogeneity of changes in risk (i.e., greater identifiability of the individuals who are benefited or harmed by a project) depends on whether the project is evaluated using compensating or equivalent variations. Using compensating variation, more information decreases WTP for risk reductions and increases WTA for risk increases, thereby decreasing the net benefits of the project. In contrast, using equivalent variation, more information increases WTA to forgo the project and decreases WTP to prevent the project, increasing the net benefits of the project. In Section 5, we examine the effects of information about heterogeneity of baseline risk. In contrast to the results for changes in risk, the effects of information about baseline risk are in general ambiguous.

Finding no systematic effect in the standard VSL model that can explain the apparent public preference for protecting identified lives, we consider other potential explanations. In Section 6, we show that altruism, defined as concern for others' well-being, does not in general justify a special concern with identified lives. In Section 7, we consider several political factors that may explain the identified-victim effect: legal responsibility or empathy for identified individuals or groups, more effective lobbying by small groups with concentrated interests, and political demagogy that appeals to voters' concern for identified lives, which may be influenced by availability or other cognitive heuristics. We conclude with a discussion of the policy implications of our analysis, which include identification of strategic motives for generation or

\footnotetext{
${ }^{6}$ Broome (1978) presents another version of the paradox based on the timing of decisions: Suppose information about who will die is not available today but will be available tomorrow. If the project is up for consideration today, it may be acceptable, but tomorrow it will be deemed unacceptable by an infinite margin. Hence re-evaluating the project one day later would lead to rejecting it as "infinitely wrong." Similarly, Heinzerling (2000) argues that the evaluation of projects that affect mortality risks should not depend on whether the lives at risk are identifiable.
} 
disclosure of information about risk heterogeneity, implications for the intertemporal consistency of BCA, and discussion of whether cost-effectiveness analysis (CEA) using life years saved or other effectiveness measures may be more consistent than BCA with maximization of social welfare.

\section{A Paradox of Benefit-Cost Analysis?}

We begin with a simple example that illustrates how the outcome of a BCA depends on information about heterogeneity of a policy-induced change in risk. Consider an economy with two people, $\mathrm{H}$ and L. Each has initial wealth $w$ and probability $p$ of surviving the single period we consider. Each is risk-neutral in wealth and has no bequest motive (i.e., the utility of wealth conditional on death is zero). Hence each individual has expected utility (EU)

$$
\mathrm{EU}=p w .
$$

Consider a project that will impose mortality risk on both people. The average incremental mortality risk is $e$. The monetary benefit of the project to each person is $s$. If people do not know their individual incremental risks (or if the incremental risks are identical) then each has expected utility

$$
\mathrm{EU} / \mathrm{no} \text { info }=(p-e)(w+s) .
$$

Defining social welfare as the mean of the two individuals' expected utilities (Harsanyi, 1953, 1955), social welfare is also given by eqn. (2).

Now consider the effect of information about individual risks. For simplicity, assume $\mathrm{H}$ (high) faces incremental risk $2 e$ and $\mathrm{L}$ (low) faces no incremental risk. Social welfare given information is

$$
\text { EU/info }=0.5(p-2 e)(w+s)+0.5 p(w+s)=(p-e)(w+s) .
$$

Social welfare with information is the same as in the no-information case. In this example, information about individual changes in risk does not affect social welfare, as Broome (1978) suggests. Moreover, observe that the project will enhance social welfare if and only if

$$
(p-e)(w+s)>p w .
$$

Consider how BCA would handle the situation. BCA involves comparing the monetary values of social benefits and costs. The per-capita benefit is $s$. The costs are the total 
compensation required to induce the people to accept the mortality risks. The compensating variation to accept a risk $x, C(x)$, is defined $b^{7}$

$$
(p-x)[w+C(x)]=p w,
$$

which implies

$$
C(x)=x w /(p-x) .
$$

In the case without information about individual risks, the per-capita cost is

$$
\mathrm{C} / \text { no info }=e w /(p-e) .
$$

With information about individual risks, the per-capita cost is

$$
\text { C/info }=0.5[2 e w /(p-2 e)]+0.5[0]=e w /(p-2 e) .
$$

Since C/info > C/no info, the decision recommended by BCA may depend on whether or not the individual risks are known. ${ }^{8}$ Specifically, if

$$
\text { C/no info }<s<\text { C/info, i.e., } e w /(p-e)<s<e w /(p-2 e),
$$

then BCA will suggest the project should proceed if there is no information about individual risks but should be rejected if individual risks are known.

Another approach to BCA is to ask if there is any lump-sum transfer $y$ such that the project with transfer is Pareto superior to rejection of the project. In the present case with information, the question is whether there is an amount $y$ that could be transferred from $\mathrm{L}$ to $\mathrm{H}$ such that $\mathrm{H}$ prefers to adopt the project, i.e.,

$$
(p-2 e)(w+s+y)>p w,
$$

and $\mathrm{L}$ prefers to adopt the project, i.e.,

$$
p(w+s-y)>p w .
$$

Eqn. (11) implies that $y<s$, i.e., L cannot provide compensation greater than $s$ if he is to prefer the project with transfer to its absence. So the maximum expected utility of $\mathrm{H}$ cannot exceed that

\footnotetext{
${ }^{7}$ Alternatively, we could consider terminal wealth with the project, $w+s$, and obtain net compensating variation, $C(x)-s$.

${ }^{8}$ Eqn. (7) demonstrates that the required compensation could be infinite for $e=p$, as Broome (1978) suggests. The example shows that this extreme case is not required to induce the type of paradox proposed by Broome.
} 
obtained when $y=s$. It is clear that eqn. (10) may not be satisfied since the survival probability $(p-2 e)$ can be arbitrarily close (or equal) to zero and so with finite $s$ the inequality is violated. ${ }^{9}$

How is it possible that information about individual risk has no effect on social welfare but may determine whether the project is adopted? The BCA criterion aggregates the monetary values of welfare gains and losses across people. The relationship between survival probability and the monetary value of utility increments can be highly non-linear. In our example, as survival probability approaches zero the monetary value of a unit of utility approaches infinity. In contrast, the expected-utility criterion aggregates increments of expected utility, which are linear in probability. Measuring in utility units, changes in individual risk (holding population risk constant) and information about individual risk have no effect on social welfare (when individuals are otherwise identical). Measuring in monetary units, information may have a large effect since the monetary value of the harm increases more rapidly for the person whose risk is increased than it decreases for the person whose risk is decreased (i.e., the indifference curve for wealth and risk and the compensation function for risk are both convex, as will be shown below).

This example illustrates a well known point: adding compensation is not the same as adding utility. ${ }^{10}$ If one wishes to use monetary compensation as an accurate proxy for adding

\footnotetext{
${ }^{9}$ Note that if one wishes to maximize social welfare it is optimal to transfer all the benefits of the project to the person with higher survival probability. That is, the maximum of $(p-2 e)(w+s+y)+p(w+s-y)$ is obtained for $y=-s$. Since there is assumed to be no bequest motive, any wealth given to a person who dies contributes no well-being to the society. This shows that social welfare can be made higher under information about individual risks than under no information. In contrast, BCA can lead to the recommendation to adopt the welfare-enhancing project only in the no-information case. Information may have a negative welfare effect for a society that uses BCA as its decision rule. Suppose the condition in eqn. (9) holds. Then if society evaluates the project using BCA, information would lead it to reject the project. Without information, the project would be accepted and social welfare would increase.

${ }^{10}$ See, e.g., Blackorby and Donaldson (1990), Johansson (1998), and in the context of mortality risk, Armantier and Treich (2004). Linerooth (1982) suggests that the higher VSL of individuals facing larger mortality risk is not sufficient justification for disproportionately allocating public resources to protect them. Although the private opportunity cost of spending is low for such individuals (by the dead-anyway effect described in Section 3), the social opportunity cost is not. Pratt and Zeckhauser (1996) propose adjusting empirical estimates of VSL to account for differences between private and social opportunity costs of spending associated with heterogeneity of mortality risk.
} 
utility, one must adjust for differences in the marginal utility of consumption between people. ${ }^{11}$ It is well known that adding unweighted compensation is valid in a first-best economy in which a social planner is able to implement lump-sum transfers to equalize marginal utility of consumption across people (Samuelson, 1954). But it is also well known that implementing optimal lump-sum transfers, or weighting compensation by the inverse of the marginal utility of consumption, confronts the problem of non-observability of utility which BCA avoids by seeking to determine only whether a proposed change is a potential Pareto improvement (i.e., satisfies a Kaldor-Hicks compensation test).

The logical point conveyed by the example is that without information individuals are identical and there is no need to weight their compensation for any difference in marginal utilities. In this case, the unweighted sum of compensation is a correct indicator of social welfare. This is not true with information about differences in individual risks. Indeed, with information the situation is asymmetric and marginal utilities may differ between individuals. Hence, whether lives are statistical or identified may affect economic valuation. In the remainder of the paper, we study the effects of individual-specific information about risks on the outcome of BCA, i.e., on the sum of unweighted individual compensation.

\section{The Value-of-Statistical-Life Model}

In the standard single-period value-of-statistical-life (VSL) model, the individual maximizes his (state-dependent) expected indirect utility given by

$$
\mathrm{V}=p u(w)+(1-p) v(w)
$$

where $p$ is the probability of surviving the period, $u(\cdot)$ is the utility of wealth if he survives the period, and $v(\cdot)$ is the utility of wealth if he dies (e.g., the utility of a bequest). This model was

\footnotetext{
${ }^{11}$ When individual costs are weighted by the individual marginal utilities of wealth, per-capita cost is independent of information about risk heterogeneity. Without information, the marginal utility of wealth is $(p-e)$ for both individuals; with information, the marginal utility of wealth is $(p-2 e)$ for $\mathrm{H}$ and $p$ for $\mathrm{L}$. With and without information, per-capita weighted cost equals $e w$. If the marginal utility of the bequest is equal to the marginal utility of wealth given survival, then the expected marginal utility is independent of risk and the monetary compensation is linear in the incremental risk (e.g., let the utility if dead equal $w-\beta$ instead of zero). In this case, maximization of social welfare and maximization of the sum of unweighted monetary compensation lead to the same outcome.
} 
introduced by Drèze (1962) ${ }^{12}$ and Jones-Lee (1974) and has been commonly used in the VSL literature (e.g., Hammitt, 2000; Viscusi and Aldy, 2003).

We adopt the standard assumptions that $u$ and $v$ are twice differentiable with

$$
u>v, u^{\prime}>v^{\prime} \geq 0, u^{\prime \prime} \leq 0 \text {, and } v^{\prime \prime} \leq 0 \text {. }
$$

That is, state-dependent utilities are increasing and weakly concave. At any wealth level both utility and marginal utility are larger if alive than dead. Under the standard assumptions (13), indifference curves over $(w, p)$ are decreasing and strictly convex as illustrated in Figure 1.

VSL is the marginal rate of substitution between wealth and survival probability, i.e., (the negative of) the slope of the indifference curve at $(w, p)$. It is defined by

$$
\mathrm{VSL}=-\frac{d w}{d p}=\frac{u(w)-v(w)}{p u^{\prime}(w)+(1-p) v^{\prime}(w)}>0 .
$$

Note that VSL may vary across individuals since it depends on $w, p$, and on the shape of the utility function through $u$ and $v$.

In the next section, we study compensating and equivalent variations in wealth for increases and decreases in risk. To first-order, these variations can be approximated by the product of VSL and the change in risk $d p .{ }^{13}$ Since VSL is a marginal rate of substitution it does not capture heterogeneity in risk change. VSL provides a good approximation to an individual's monetary compensation for a change in risk only when the change is sufficiently small. At the population level, average VSL provides a good approximation to the social value of a change in population risk if all risk changes in the population are small. ${ }^{14} \mathrm{~A}$ formally equivalent situation is

\footnotetext{
${ }^{12}$ Published in French, Drèze's paper is not well known among Anglophone economists although it was discussed by Jones-Lee $(1974,1976)$. Drèze modeled individual WTP to eliminate a onetime mortality risk and optimal spending on risk reduction using the now-standard statedependent utility function (12).

${ }^{13}$ Formally, VSL is equal to the marginal compensating and equivalent variations $C^{\prime}(0), P^{\prime}(0)$, $C_{n}$ '(0), and $P_{n}{ }^{\prime}(0)$, that are defined below in eqns. (16), (18), (20) and (23), respectively. JonesLee (1976) also evaluated these four measures of value.

${ }^{14}$ The term value per statistical life may be motivated by noting that if a population of $N$ individuals would each pay on average $d w$ for a small risk reduction $d p$, a total of $N d w$ would be paid to prevent $N d p$ expected fatalities.
} 
that of a few individuals facing a large change in risk when these individuals are anonymous (to themselves and to others). ${ }^{15}$

The expression for VSL in eqn. (14) is useful for identifying two effects that will be important for our analysis. First, the dead-anyway effect (Pratt and Zeckhauser, 1996) describes how VSL increases with risk, i.e., decreases with survival probability $p$ (i.e., $\partial \mathrm{VSL} / \partial p<0$ ). In Figure 1, the indifference curve is steeper at B than at A. Intuitively, an individual facing a large total probability of death has little incentive to limit his spending on risk reduction since he is unlikely to survive and have other opportunities for consumption. In eqn. (14), the value of the numerator is independent of $p$ and a decrease in $p$ reduces the value of the denominator (since $\left.u^{\prime}>v^{\prime}\right) .{ }^{16}$ Second, the wealth effect describes how VSL increases with wealth $w$, i.e., $\partial \mathrm{VSL} / \partial w>0$ and the indifference curve in Figure 1 is steeper at $\mathrm{C}$ than at $\mathrm{A}$. The intuition for the wealth effect is two-fold. First, wealthier people have more to loose if they die (i.e., the numerator in eqn. (14) increases with $w$ because $u^{\prime}>v^{\prime}$ ). Second, the utility cost of spending is smaller (or no larger) due to weakly diminishing marginal utility (risk aversion) with respect to wealth (i.e., the denominator in eqn. (14) does not increase because $u " \leq 0$ and $v^{\prime \prime} \leq 0$ ). Hence, VSL increases as one moves upward and leftward along an indifference curve and the indifference curves are convex. In Figure 1, VSL at D is greater than VSL at A because of both the dead-anyway and the wealth effects.

\footnotetext{
${ }^{15}$ Identifiability may impair rational decision making when the threat of death is present. This is recognized by Schelling (1968): "The avoidance of a particular death - the death of a named individual- cannot be treated straightforwardly as a consumer choice. It involves anxiety and sentiment, guilt and awe, responsibility and religion. If the individuals are identified, there are many of us who cannot even answer whether one should die that two may live.” We abstract from this issue but recognize it may play an important role for practical BCA, e.g. for elicitation of individual WTP in surveys. Observe however that opposite arguments may be used with statistical lives due to the small probabilities involved, like the insensitivity of WTP to probabilities (Hammitt and Graham, 1999).

${ }^{16}$ The dead-anyway effect was recognized by Jones-Lee (1974, 1976), Weinstein et al. (1980), Linerooth (1982), and others but the moniker was bestowed by Pratt and Zeckhauser (1996). It depends on $u^{\prime}>v^{\prime}$, a condition that may not hold with perfect contingent-claims markets (JonesLee, 1976; Weinstein et al., 1980; Breyer and Felder, 2005) or when desired bequests are constrained because human capital or other components of wealth do not survive the individual's death (Breyer and Felder, 2005).
} 


\section{Information about Heterogeneous Changes in Risk}

The example in Section 2 shows that information about risk heterogeneity can affect a policy decision that is based on BCA. The strength of the effect depends on the non-linearity of the compensation function $C(x)=x w /(p-x)$ (eqn. (6)). The crucial property is the convexity of this function, which we now demonstrate.

Consider two individual-specific risk increments, $x_{1}$ and $x_{2}$. In the absence of information about individual-specific risk, each individual faces an expected risk increment of $\bar{x}=0.5 x_{1}+0.5 x_{2}$ and requires compensation $C(\bar{x})$ (see Figure 2). Under perfect information, each knows his specific incremental risk and average compensation is $0.5 C\left(x_{1}\right)+0.5 C\left(x_{2}\right)$. Information increases average compensation iff $0.5 C\left(x_{1}\right)+0.5 C\left(x_{2}\right) \geq C(\bar{x})$, i.e., when $C(x)$ is convex. Clearly, convexity of the compensation function is a necessary and sufficient condition for information about individual-specific risk changes to increase average compensation for any distribution of individual-specific risk changes in the economy (holding the average risk change fixed). Moreover, convexity of the indifference curves over $(w, p)$ implies that $C(x)$ is convex.

Analyzing the convexity of $C$ is equivalent to comparing the compensation required for successive risk increments. Compare the compensation required for an incremental risk increase of $x$ to the compensation required for a second equal increment $x$ once the first has been made. Convexity implies that compensation for the second increment is larger than for the first. There are two differences in the compensation for the second unit compared with the first: i) survival probability is lower, $p-x$ compared with $p$; and ii) wealth is higher, $w+C(x)$ compared with $w$. The first difference increases compensation through the dead-anyway effect and the second increases compensation through the wealth effect.

In the remainder of this section we use the standard VSL model (14) to study how information affects the valuation of changes in risk. As we have just seen, this amounts to studying the convexity of the compensation functions for changes in risk. We consider the valuation of both increases and decreases in risk, using both WTA and WTP. We find that the conclusions differ in each case. 


\subsection{WTA for implementing a risk-increasing project}

Generalizing the introductory example, we first consider WTA for a risk-increasing project. Let $C(x)$ be the WTA (compensating variation) defined by

$$
(p-x) u(w+C(x))+(1-p+x) v(w+C(x))=p u(w)+(1-p) v(w)
$$

with $0 \leq x \leq p$. Differentiating with respect to $x$ gives

$$
C^{\prime}(x)=\frac{u(w+C(x))-v(w+C(x))}{(p-x) u^{\prime}(w+C(x))+(1-p+x) v^{\prime}(w+C(x))}>0 .
$$

Differentiating once again with respect to $x$ gives, under our assumptions on $u$ and $v, C^{\prime \prime}>0$. As noted above, the convexity of $C$ is immediate from the convexity of the indifference curve. Indeed, we have $C(\bar{x})<0.5\left[C\left(x_{1}\right)+C\left(x_{2}\right)\right]$ (see Figure 2). Since WTA is convex in risk increments it increases with information about individual risk changes. This is due to the deadanyway effect and the wealth effect, both of which are positive in this case, as shown above.

This case shows that information about individual risk changes increases the cost of the risk-increasing project and induces a bias in favor of the status quo. With more information, a project that would have been accepted may be rejected, preventing an increase in mortality risks in the economy. This is the case illustrated by Broome's (1978) paradox.

\subsection{WTP for implementing a risk-decreasing project}

Let $P(x)$ be the WTP (compensating variation) for implementing a risk-decreasing project defined by

$$
(p+x) u(w-P(x))+(1-p-x) v(w-P(x))=p u(w)+(1-p) v(w)
$$

with $0 \leq x \leq 1-p$. Differentiating with respect to $x$ gives

$$
P^{\prime}(x)=\frac{u(w-P(x))-v(w-P(x))}{(p+x) u^{\prime}(w-P(x))+(1-p-x) v^{\prime}(w-P(x))}>0 .
$$

Differentiating once again with respect to $x$ gives $P^{\prime \prime}<0$. Observe from Figure 2 that this result is immediate from the convexity of the indifference curve, i.e., $P(\bar{x})>0.5\left[P\left(x_{1}\right)+P\left(x_{2}\right)\right]$. WTP is concave in risk reductions and thus decreases with information about individual risk changes. Both the dead-anyway and wealth effects are negative in this case.

This case shows that more information decreases the benefit of the risk-decreasing project and, like the previous case, induces a bias in favor of the status quo. More information may lead 
to rejecting a project that would have been accepted, forgoing an opportunity to reduce mortality risks in the economy.

Combining the results from Sections 4.1 and 4.2 implies that more information about risk heterogeneity has a predictable effect on BCA. When using compensating variation (i.e., WTP to reduce risk and WTA compensation for a risk increase), more information about risk heterogeneity decreases the benefits and increase the costs, and hence discourages adoption, of a project that decreases risk to some and increases risk to other members of the economy. ${ }^{17}$

\subsection{WTA for not implementing a risk-decreasing project}

Let $C_{n}(x)$ be the WTA (equivalent variation) for forgoing a risk-decreasing project defined by

$$
p u\left(w+C_{n}(x)\right)+(1-p) v\left(w+C_{n}(x)\right)=(p+x) u(w)+(1-p-x) v(w)
$$

with $0 \leq x \leq 1-p$. Differentiating with respect to $x$ gives

$$
C_{n}^{\prime}(x)=\frac{u(w)-v(w)}{p u{ }^{\prime}\left(w+C_{n}(x)\right)+(1-p) v^{\prime}\left(w+C_{n}(x)\right)}>0 .
$$

Differentiating once again with respect to $x$ gives

$$
C_{n}{ }^{\prime \prime}(x)=-\frac{\left(C_{n}^{\prime}(x)\right)^{2}\left[p u^{\prime \prime}\left(w+C_{n}(x)\right)+(1-p) v^{\prime \prime}\left(w+C_{n}(x)\right)\right]}{p u^{\prime}\left(w+C_{n}(x)\right)+(1-p) v^{\prime}\left(w+C_{n}(x)\right)} .
$$

Under our assumptions on $u$ and $v, C_{n} " \geq 0$. WTA is convex and thus increases with information. In Figure 2, $C_{n}(\bar{x}) \leq 0.5\left[C_{n}\left(x_{1}\right)+C_{n}\left(x_{2}\right)\right]$. Observe that under risk neutrality (i.e., $\left.u "=v^{\prime \prime}=0\right), C_{n}$ is linear and there is no effect of information. Under strict risk aversion (i.e., $u "<0$ and $v^{\prime \prime}<0$ ), the dead-anyway effect is negative and the wealth effect is positive, suggesting that the latter effect dominates. Note that it is not possible a priori to determine the convexity of $C_{n}$ from Figure 2.

This case shows that more information increases the benefit of the risk-decreasing project and induces a bias in favor of the status quo (implementing the project). In this case, more

\footnotetext{
${ }^{17}$ Note that the results rely on only the convexity of the indifference curves and that the conditions exhibited in (13) are sufficient, but not necessary, for this convexity property to hold. This suggests broader applications of the results than to pure mortality risks.
} 
information may lead to adopting a project that would have been rejected, reducing mortality risks in the economy.

\subsection{WTP for not implementing a risk-increasing project}

Let $P_{n}(x)$ be the WTP (equivalent variation) to prevent implementing a risk-increasing project defined by

$$
p u\left(w-P_{n}(x)\right)+(1-p) v\left(w-P_{n}(x)\right)=(p-x) u(w)+(1-p+x) v(w)
$$

with $0 \leq x \leq p$. Differentiating with respect to $x$ gives

$$
P_{n}^{\prime}(x)=\frac{u(w)-v(w)}{p u{ }^{\prime}\left(w-P_{n}(x)\right)+(1-p) v^{\prime}\left(w-P_{n}(x)\right)}>0 .
$$

Differentiating once again with respect to $x$ gives

$$
P_{n}^{\prime \prime}(x)=\frac{\left(P_{n}^{\prime}(x)\right)^{2}\left[p u^{\prime \prime}\left(w-P_{n}(x)\right)+(1-p) v^{\prime \prime}\left(w-P_{n}(x)\right)\right]}{p u^{\prime}\left(w-P_{n}(x)\right)+(1-p) v^{\prime}\left(w-P_{n}(x)\right)} .
$$

Under our assumptions on $u$ and $v, P_{n} " \leq 0$. WTP is concave and decreases with information. In

Figure 2, $P_{n}(\bar{x}) \geq 0.5\left[P_{n}\left(x_{1}\right)+P_{n}\left(x_{2}\right)\right]$. Note that, as for the previous case, there is no effect under risk neutrality and under strict risk aversion the wealth effect (which is negative) dominates the dead-anyway effect (which is positive). Note again that it is not possible a priori to infer the sign of the second derivative of $P_{n}$ from Figure 2.

This case shows that more information decreases the cost of the risk-increasing project and induces a bias in favor of the status quo (implementing the policy). More information may lead to adopting a project that would have been rejected and thus increase mortality risks in the economy.

Combining results from Sections 4.3 and 4.4 implies that when BCA is conducted using equivalent variation measures (i.e., WTA to forgo a risk reduction, WTP to prevent a risk increase), information about risk heterogeneity increases WTA, decreases WTP, and discourages abandonment of a project that decreases risk to some and increases risk to others.

\section{Information about Heterogeneous Baseline Risks}

In Section 4, the initial risk is common across individuals although the risk changes can differ. In this section we consider how information about heterogeneity of baseline risk can affect 
valuation. We begin with a problem first analyzed by Pratt and Zeckhauser (1996) on the concentration of risk in a community, in which the baseline risk and risk change are perfectly correlated in the population. We then consider a simpler problem in which baseline risk is heterogeneous but the policy-induced risk change is common across the population. We restrict our analysis to compensating variation (WTP for a risk reduction, WTA for a risk increase) and find that the effect of information is generally ambiguous when individuals are risk averse.

\subsection{Heterogeneity in both change and baseline risk}

Suppose there are $N$ people in the economy each with initial wealth $w$. Only $R \leq N$ people are at risk and of these $R$ people $d \leq R$ will die. None of the $N-R$ others will die. A reduction in $R$ may be interpreted as both a change in the concentration of risk in the population and as a change in individual-specific information in the economy.

We return to the simplest possible assumptions as in our initial example, i.e., $u(z)=z$ and $v(z)=0$, which we loosely describe as the risk-neutral case. Social welfare in this economy equals

$$
(N-R) w+R \frac{R-d}{R} w=(N-d) w .
$$

Observe that social welfare is independent of $R$. Suppose that a risk-reducing project will save $n$ lives $(n \leq d)$ for a given per-capita cost $F$. The net effect of the project on social welfare is also independent of $R$.

Let us now look at how BCA would handle the situation. Let $P(R)$ be the WTP (compensating variation) for the project of each of the $R$ people who benefit, defined by

$$
\frac{R-d+n}{R}[w-P(R)]=\frac{R-d}{R} w,
$$

which yields

$$
P(R)=\frac{n}{R-d+n} w .
$$

Both individual WTP $P(R)$ and total benefits $R P(R)$ are decreasing in $R$. Information about risk heterogeneity affects the outcome of BCA but not social welfare.

Note the direction of the bias: the monetary value of the benefit of the project is larger when the number of people who are identified as at risk is smaller, i.e., a "benefit-concentration" 
effect. This result is opposite the one obtained in Section 4.2 where aggregate WTP decreases with information about individual risk reductions. Here information about which people face larger risk reductions increases aggregate WTP. Better information about individual-specific risks may lead to adopting a project that would otherwise be rejected, decreasing mortality risk in the economy.

Alternatively, we can evaluate WTA (compensating variation) for a risk-increasing project. Suppose that a risk-increasing project will kill $r$ people in this economy $(r \leq R-d)$ for some social benefit. Let $C(R)$ be defined by

$$
\frac{R-d-r}{R}[w+C(R)]=\frac{R-d}{R} w
$$

which yields

$$
C(R)=\frac{r}{R-d-r} w .
$$

Individual WTA denoted $C(R)$ decreases with $R$ as does the total social cost $R C(R)$. In this case, better information as to which people face additional risk increases aggregate WTA. Better information may lead to rejecting a project that would otherwise be accepted, preventing an increase in mortality risk as in Broome’s (1978) paradox.

To summarize, under risk neutrality with respect to wealth, more information about the concentration of risk and risk change can lead to lower mortality risks in the economy. This can occur through either of two pathways: adopting risk-reducing projects that would be rejected and rejecting risk-increasing projects that would be adopted with poorer information about the concentration of risks and benefits.

What is the effect of a change in $R$ in a model with risk aversion? Consider the following example. Take the utility function

$$
u(z)=\sqrt{z} \text { and } v(z)=0
$$

together with the following set of parameter values: $w=1, d=50$, and $n=20$. It is straightforward to obtain

$$
P(R)=w\left[1-\left(\frac{R-d}{R-d+n}\right)^{2}\right]=\frac{40(R-40)}{(R-30)^{2}}
$$

so that $R P(R)$ is increasing over $R \in[50,60]$ and decreasing over $R>60$. In this case information about how many people are at risk has an ambiguous effect on aggregate WTP. 
The non-monotonicity of $R P(R)$ reflects the competition between two effects: as $R$ decreases, fewer people will pay but each is willing to pay more. This example proves that is not possible a priori to determine which effect dominates under risk-averse preferences, as previously shown by Pratt and Zeckhauser (1996: 753).

\subsection{Heterogeneity in baseline risk}

The previous result suggests that the effect of information about the concentration of risk in an economy is more difficult to sign than the effect of information about differences in risk changes analyzed in Section 4. This can be easily understood. In the case of information about which people are at risk there are not one but two sources of individual-specific risks: risk over the baseline probability and risk over the change in probability. In the cases considered in Section 5.1, these risks are perfectly positively correlated.

We next consider the simpler problem of analyzing the effect of information about only heterogenous baseline risks. Assume that people have different survival probabilities $p$ but the risk reduction $x$ is the same for everyone. We can show that information about baseline risks has an ambiguous effect in this case as well. Take the same risk-averse utility function as in (30). Compute WTP to reduce risk by $x$ (now denoted $P(p)$ ) as in eqn. (17) to obtain

$$
P(p)=\frac{w x(2 p+x)}{(p+x)^{2}} .
$$

From Section 4.2 it is easy to understand that the convexity of $P(p)$ in $p$ determines the effect of information about baseline probability on aggregate WTP. Yet from eqn. (32) the sign of this effect is ambiguous as the sign of $P^{\prime \prime}(p)$ depends on the sign of $(p-x / 2)$. Hence the effect of more information about heterogeneous baseline risk depends on the values of $p$ and $x$. Again, it is easy to see that this ambiguity disappears under risk-neutrality, in the sense that more information about baseline probabilities increases aggregate WTP and aggregate WTA for all values of $p, x$ and $w$.

In contrast to the effects of information about heterogeneity of risk reduction analyzed in Section 4, this section shows that the effects of information about heterogeneity in baseline risk on the value of risk changes are in general ambiguous. This ambiguity results when differences in risk changes are perfectly correlated with differences in baseline risk and also when risk changes 
are common across individuals. An exception occurs for the case of risk neutrality with respect to wealth where information about either risk concentration or heterogeneous baseline risks increases both aggregate WTP for a risk-reducing program and aggregate WTA for a riskincreasing program.

\section{Altruism and the Identifiable-Victim Effect}

In this section, we briefly discuss the effect of altruism. Concern for others' well being is an important and natural concern in the matter of saving lives. Moreover, it is widely believed that there is an "identifiable-victim effect": people are willing to pay more to save the lives of others when these lives are easily identifiable than when they are statistical (see, e.g., Jenni and Loewenstein, 1997; Heinzerling, 2000; Small and Loewenstein, 2003). Typically, there is an empathic response to rescuing identified people (or animals) in precarious circumstances. There is little hope of capturing all the channels underlying such empathic responses in a simple economic model (recall the quote from Schelling (1968) in footnote 15). In any case, our previous model did not have a chance to do so since there was no consideration of altruism. ${ }^{18}$

We consider only the case of pure altruism. ${ }^{19}$ It has been shown that pure altruism may be ignored in BCA. The sum of private compensating variations is indeed an adequate estimate of the benefits of the project in the sense that if this sum exceeds the sum of the private costs of the project, then the project is a potential Pareto improvement for altruistic people. This point was first stressed by Bergstrom (1982), elaborated by Jones-Lee (1992), and generalized by Bergstrom (2006). The argument is simple. A pure altruist benefits when the project reduces another person's risk but is harmed when the project imposes costs on the other person. The sign of one person's altruistic valuation for another is the same as the sign of the net private benefits to the other and so pure altruism cannot alter the sign of the social net benefits.

\footnotetext{
${ }^{18}$ In model (12), a natural interpretation of the utility function $v(\cdot)$ is as a bequest function. This may be viewed as a form of altruism, e.g., toward one's children. But this is not the form of altruism that is important here. We study altruistic preferences over other people's mortality risks.

${ }^{19}$ See Jones-Lee (1992) for an analysis of the implications of both pure (i.e., benevolent, nonpaternalistic) and paternalistic altruism (e.g., safety-focused altruism) for the valuation of life.
} 
The implication of this observation is that pure altruism should not affect the valuation of lives in BCA, no matter how much information is available about risk heterogeneity, and hence pure altruism cannot justify an identifiable-victim effect.

\section{Policy Implications}

A standard economic result is that public projects with positive expected financial returns should be adopted (Arrow and Lind, 1970), no matter how risk averse are individuals. This conclusion assumes an economy with either complete markets or a large number of individuals who can share financial risks. In this economy, the sum of individual compensating variations for a project is consistent with the maximization of social welfare, with or without information on the distribution of the project's effects among individuals, because complete markets for risk-sharing yield an efficient outcome at which individuals equate their marginal utilities. When risk-sharing opportunities are limited, the rule of acceptance for public projects is less clear and the adequacy of BCA as a method for identifying gains in social welfare is not guaranteed.

An important case of limited risk-sharing is when a bad outcome decreases marginal utility, a property of the standard state-dependent model for valuing mortality risk described by expressions (12) and (13). In this case, it may be that some individuals could never be compensated for their loss, as suggested by Broome (1978). Marginal utilities may differ among people and BCA may not be a correct indicator of social welfare. Individual-specific information about mortality risks - whether lives are statistical or identified - may affect the result of BCA.

We have studied some theoretical determinants of this difference. Let us summarize some results:

1. The effects of information about interpersonal differences in the risk change due to a project depend on whether one evaluates the project using WTP or WTA measures.

a. WTA, whether compensating variation for a risk increase (Section 4.1) or equivalent variation to forgo a risk decrease (Section 4.3), is larger with more information about heterogeneity of the risk change. Information about how the project affects individuals' risks may lead to rejecting a risk-increasing project that would have been accepted (as in Broome’s paradox) or accepting a risk-decreasing project that would have been rejected with less information. In both cases, information about heterogeneity leads toward lower aggregate risk in the economy. 
b. WTP, whether compensating variation for a risk decrease (Section 4.2) or equivalent variation to prevent a risk increase (Section 4.4), is smaller with more information about heterogeneity of the risk change. Information about how the project affects individuals' risks may lead to rejecting a risk-decreasing project that would have been accepted and accepting a risk-increasing project that would have been rejected with less information. In both cases, information about heterogeneity leads toward higher aggregate risk in the economy.

c. As a corollary, there is no necessary relationship between the extent to which risk changes are statistical or identifiable and the degree of protection endorsed by BCA.

2. When baseline risks are heterogeneous, the effect of information on BCA is in general ambiguous. When individuals are risk neutral with respect to wealth more information about baseline risk can lead to increases in both types of compensating variation, i.e., WTP for a risk-decreasing policy and WTA for a risk-increasing policy, and can therefore lead to more protective decisions, yielding smaller aggregate risk.

3. BCA does not explain the identifiable-victim effect, even with pure altruism. This is a consequence of a more general result that pure altruism need not be considered in BCA (Bergstrom, 2006).

It is interesting to compare BCA with cost-effectiveness analysis (CEA). In CEA, changes in health risk are often evaluated by the expected change in lives, life years, or quality-adjusted life years (QALYs) in the economy. ${ }^{20}$ Evaluation of the effects of a program on mortality risk is unaffected by whether lives are statistical or identified or by information about heterogeneity of baseline risks or risk changes (holding constant population risk change as measured by the measure of effectiveness that is employed). This result is immediate since the expected change in effectiveness is linear in the probabilities. If individuals' preferences for survival are consistent with expected life years or QALYs, CEA using these measures may be consistent with social

${ }^{20}$ A QALY represents a year of life adjusted by an index of quality of life or health (Gold et al., 1996; see Zeckhauser and Shepard (1976), Broome (1993), and Hammitt (2002) for economic discussions). The US Office of Management and Budget (2003) requires federal agencies to evaluate major regulations using CEA as well as BCA and an Institute of Medicine panel recommended that QALYs be used as the measure of effectiveness in this context (Miller et al., 2006). 
welfare maximization (Hammitt, 2002; Adler, 2006). ${ }^{21}$ Hadorn (1996) reports that the insensitivity of the QALY approach to heterogeneity was criticized in the famous Oregon priority-setting exercise. The approach was perceived as yielding an ordering that undermined treatments for people facing life-threatening conditions, thus violating a preference for giving priority to identified victims. ${ }^{22}$

Overall, neither BCA nor CEA provide general support for prioritizing identified over anonymous lives in life-saving policies. Political factors are, however, natural candidates to explain such a prioritization.

\subsection{Political factors}

We discuss four political factors that may explain why statistical and identified lives are treated differently: responsibility, empathy, lobbying, and demagogy. ${ }^{23}$

First, it is important to recognize that persons with authority for safety decisions may have a responsibility to help identified people. In particular, there may be a legal responsibility. ${ }^{24} \mathrm{By}$ definition, the death of a victim identified ex post will be known and blame may be easily attributed to decision makers whereas the deaths of ex post anonymous individuals may be unnoticed or more difficult to attribute to particular decisions (Raiffa et al., 1977). Risk managers routinely set limits on the risks to which individuals may be exposed, often focusing on absolute risk levels (e.g., safety norms or standards). This practice may lead to protecting only highly exposed individuals and thus toward ex ante identifiable individuals. Notice that ex ante identification need not be restricted to those exposed to high risk. Providing greater protection to

${ }^{21}$ Empirical evidence suggests that QALYs are not consistent with individual preferences. See, e.g., Pliskin et al. (1980), McNeil et al. (1981), and Bleichrodt and Johannesson (1994).

${ }^{22}$ Richardson and McKie (2003) give as an example that "dental caps for pulp or near pulp exposure were assigned a higher priority than surgical treatment for ectopic pregnancy." Miller et al. (2006) evaluate the implications of QALYs for allocating risk reduction in a population.

${ }^{23}$ Fernandez and Rodrik (1991) show that individual-specific uncertainty may change the identity of the median voter and hence the projects that will be selected by majority vote. We do not address how this effect might be influenced by information about heterogeneity of risk.

${ }^{24}$ Richardson and McKie (2003), quoting Fried (1969: 1418), indicate that the director of a mining company refusing to spend money to save a trapped man may be civilly and criminally liable even if she has invested large sums of money to prevent such a catastrophe. Similar legal responsibilities may exist in hospitals, e.g., to care for terminally ill patients. 
members of a minority or low-income group is a way to compensate them for other disadvantages, ${ }^{25}$ consistent with environmental justice (Tietenberg, 1996).

Second, decision makers may believe they have a special duty to help identified groups. Almost by definition, identified persons will be more salient to a decision maker who must target a specific policy objective. Empathy and vividness may also contribute. In contrast, decision makers may not appreciate that preoccupation with identified lives may contribute to "statistical murder.” They may prefer to base their policies on intuitive or emotional criteria rather than on BCA or CEA. They may be more willing to endorse perceived humane and caring policies and less willing to adopt policies that they perceive as unfair or unethical toward particular groups. This posture may be supported by their education or political agenda.

Third, one may view society as composed of different groups of people, or lobbies, sharing common interests. Participants in small lobbies may have more intense interests and free riding may be less pronounced, yielding disproportionate political influence (Olson, 1971). A policy that favors concentrated interests may be more likely to be adopted than a policy that profits the mass but adversely affects a few. Similarly, the existence of a group that is particularly visible, e.g., through media exposure, may constitute a threat to politicians or firms with concerns about reputation. Visibility of a group may also be used to inform or manipulate the public. This practice may exert an indirect pressure on decision makers through ensuing changes in public beliefs (Kuran and Sunstein, 1999).

The last remark leads us to a fourth political factor, demagogy of politicians. Politicians might adopt policies that the public believes are good even when those policies are not necessarily good for the public. The public may prefer to reduce risk to identified victims because these induce a stronger emotional response (Jenni and Lowenstein, 1997). Identifying victims of a particular risk may also make that risk more salient and increase concern about one’s own exposure to it through the availability heuristic (Tversky and Kahneman, 1974), increasing demand for mitigation (Sunstein, 2005). This aspect may be aggravated if individuals

${ }^{25}$ This practice is critically discussed by Viscusi (2000b). Note that a simple model of public provision of safety would actually predict quite an opposite result. According to this model, it is optimal to devote more resources to prevent risks faced by the sub-population of, say, richer people. Such a practice is efficient since it rests on the assumption that the government is also able to implement lump-sum transfers to redistribute income and finance the safety provision across the population. Richer people will be taxed more heavily to compensate poorer people so that, at the optimum, marginal utilities are equal. 
misperceive the (marginal) costs of government services or favor government allocations based on ethical principles like equality (Kemp, 2003).

\subsection{Policy use of benefit-cost analysis}

We conclude by summarizing some remarks and raising additional concerns about the use of BCA for policy decisions.

BCA using the standard VSL approach for valuing changes in mortality risk may not yield the same results as maximization of social welfare, defined as the mean of individual expected utilities. In particular, whereas social welfare may be unaffected by information about heterogeneity of baseline risk and of the magnitudes of policy-induced changes in risk, monetary measures of the risk change depend on baseline risk and risk change and so the value of a policyinduced risk change may depend on information about risk heterogeneity. In contrast, the results of a cost-effectiveness analysis (CEA), which quantifies the effects of changes in mortality risk using lives saved, quality-adjusted life years, or other metrics, may be unaffected by information about risk heterogeneity. Hence if the effectiveness measure used in CEA is consistent with individuals' preferences, the results of the analysis may be consistent with social-welfare maximization.

Our results suggest there may be more opportunity for discretion and strategy in applying BCA as a policy tool when there is greater risk heterogeneity. Suppose that some actors concerned about a project possess information about risk heterogeneity. One can imagine that they may wish to release this information strategically so that the adoption rule matches their policy interest. The strategic value of information also provides incentives to obtain it. Similarly, when information is public and arrives exogenously, opportunistic actors may wish to accelerate or delay the decision-making process.

Our findings may be useful in evaluating the intertemporal consistency of decisions based on BCA. An initial BCA may recommend adopting a project but additional information concerning unequal distribution of risk may reverse the recommendation. Information about heterogeneity of risk change usually favors the status quo. This suggests that an initial decision about whether to implement a project is unlikely to be reversed by a subsequent BCA conducted when more information is available, taking the early decision as the new status quo. This inertia, although reducing the possibility of Scitovsky switchings over time, may be socially inefficient. 
CEA, which is usually unaffected by information about risk heterogeneity, may be less susceptible to such socially inefficient manipulations.

A systematic premium for identified lives is not consistent with either BCA or CEA but may be explained by political and other factors such as symbolic support for individuals. Whether disproportionate allocation of resources toward programs that save identified lives is socially desirable depends in part on the moral legitimacy of those factors and on the magnitudes of the efficiency costs and other benefits. 


\section{References}

Adler, Matthew D., 2006, QALYs and policy evaluation: a new perspective, Yale Journal of Health Policy, Law, and Ethics, 6, 1-92.

Armantier, Olivier, and Nicolas Treich, 2004, Social willingness-to-pay, mortality risks and contingent valuation, Journal of Risk and Uncertainty 29, 7-19.

Arrow, Kenneth J., and Robert Lind, 1970, Uncertainty and the evaluation of public investment decisions, American Economic Review 1, 364-78.

Bergstrom, Theodore, 1982, When is a man's life worth more than his human capital? The Value of Life and Safety (M. Jones-Lee, ed.), 3-26, North-Holland, Amsterdam.

Bergstrom, Theodore, 2006, Benefit-cost in a benevolent society, American Economic Review 96, 339-351.

Blackorby, Charles, and David Donaldson, 1990, A review article: the case against the use of the sum of compensating variations in benefit-cost analysis, Canadian Journal of Economics 23, 471-94.

Bleichrodt, Han, and Magnus Johannesson, 1996, The validity of QALYs: an experimental test of constant proportional tradeoff and utility independence, Medical Decision Making 17, 2132.

Breyer, Friedrich and Stefan Felder, 2005, Mortality risk and the value of a statistical life: the dead-anyway effect revis(it)ed, The Geneva Papers on Risk and Insurance Theory 30, 4155 .

Broome, John, 1978, Trying to value a life, Journal of Public Economics 9, 91-100.

Broome, John, 1993, Qalys, Journal of Public Economics 50, 149-67.

Buchanan, James M., and Roger L. Faith, 1979, Trying again to value a life, Journal of Public Economics 10, 245-48.

Corso, Phaedra S., James K. Hammitt, John D. Graham, Richard C. Dicker, and Sue J. Goldie, 2002 Assessing preferences for prevention versus treatment using willingness to pay, Medical Decision Making 22, S92-S101.

Drèze, Jacques, 1962, L'utilité sociale d'une vie humaine, Revue Française de Recherche Opérationnelle 6, 93-118.

Fernandez, Raquel, and Dani Rodrik, 1991, Resistance to reform: status quo bias in the presence of individual-specific uncertainty, American Economic Review 81, 1146-55.

Fried, Charles, 1969, The value of life, Harvard Law Review 82, 1415-37.

Gold, Marthe R., Joanne E. Siegel, Louise B. Russell, and Milton C. Weinstein, 1996, CostEffectiveness in Health and Medicine, Oxford University Press, Oxford.

Graham, John D., 1995, Comparing Opportunities to Reduce Health Risks: Toxin Control, Medicine, and Injury Prevention, National Center for Public Administration Policy Report No. 192, Dallas. 
Hadorn David C., 1996, The Oregon-priority-setting exercise: cost-effectiveness and the rule of rescue revisited, Medical Decision Making 16, 117-19.

Hammitt, James K., 2000, Valuing mortality risk: theory and practice, Environmental Science and Technology 34, 1396-1400.

Hammitt, James K., 2002, QALYs versus WTP, Risk Analysis 22, 985-1001.

Hammitt, James K., and John D. Graham, 1999, Willingness to pay for health protection: inadequate sensitivity to probability, Journal of Risk and Uncertainty 3, 33-62.

Harsanyi, John C., 1953, "Cardinal utility in welfare economics and in the theory of risk-taking," Journal of Political Economy 61, 434-435.

Harsanyi, John C., 1955, "Cardinal welfare, individualistic ethics, and interpersonal comparisons of utility,” Journal of Political Economy 63, 309-321.

Heinzerling, Lisa, 2000, The rights of statistical people, Harvard Environmental Law Review 24, 189-207.

Howard, Ron A., 1984, On fates comparable to death, Management Science 30, 407-422.

Hsu, Shi-Ling, 2007, The identifiability bias in environmental law, mimeo, University of British Columbia Faculty of Law.

Jenni, Karen E., and George Loewenstein, 1997, Explaining the "identifiable victim effect”, Journal of Risk and Uncertainty 14, 235-257.

Johansson, Per-Olov, 1998, Does the choice of numéraire matter in benefit-cost analysis? Journal of Public Economics 70, 489-93.

Jones-Lee, Michael W., 1974, The value of changes in the probability of death or injury, Journal of Political Economy 99, 835-849.

Jones-Lee, Michael W., 1976, The Value of Life: An Economic Analysis, University of Chicago Press, Chicago.

Jones-Lee, Michael W., 1979, Trying to value a life: why Broome does not sweep clean, Journal of Public Economics 10, 249-56.

Jones-Lee, Michael W., 1992, Paternalistic altruism and the value of statistical life, Economic Journal 102, 80-90.

Jones-Lee, Michael W., 2004, Valuing international safety externalities: does the 'golden rule' apply? Journal of Risk and Uncertainty 29, 277-287.

Keeney, Ralph L., 1995, Understanding life-threatening risks, Risk Analysis 15, 627-637.

Kemp, Simon, 2003, Public Goods and Private Wants: A Psychological Approach to Government Spending, Edward Edgar, Northampton, Massachusetts.

Kuran, Timor, and Cass R. Sunstein, 1999, Availability cascades and risk regulation, Stanford Law Review 51, 683-768.

Lamm, Richard D., 2001, Compassion for unidentified lives, Healthplan 42, May/June.

Linnerooth, Joanne, 1982, Murdering statistical lives. The Value of Life and Safety (M. JonesLee, ed.), 229-261, North-Holland, Amsterdam. 
McNeil, Barbara J., Ralph Weichselbaum, and Steven G. Pauker, 1981, Speech and survival: tradeoffs between quantity and quality of life in laryngeal cancer, New England Journal of Medicine 305, 982-987.

Merrell, P. and C. Van Strum, 1990, Negligible risk: premeditated murder? Journal of Pesticide Reform 10, 20-22.

Miller, Wilhelmine, Lisa A. Robinson, and Robert S. Lawrence (eds.), 2006, Valuing Health for Regulatory Cost-Effectiveness Analysis, Institute of Medicine, National Academies Press, Washington, D.C.

Mishan, Ezra J., 1971, Evaluation of life and limb: a theoretical approach, Journal of Political Economy 79, 687-705.

Okun, Arthur M, 1975, Equality and Efficiency: The Big Tradeoff. The Brookings Institution, Washington, DC.

Olson, Mancur, 1971, The Logic of Collective Action: Public Goods and the Theory of Groups, Harvard University Press, Cambridge.

Pliskin, Joseph S., Donald S. Shepard, and Milton C. Weinstein, 1980, Utility functions for life years and health status, Operations Research 28, 206-224.

Pratt, John W., and Richard J. Zeckhauser, 1996, Willingness to pay and the distribution of risk and wealth, Journal of Political Economy 104, 747-63.

Raiffa, Howard, Schwartz William B. and Milton C. Weinstein, 1977, Evaluating health effects of societal decisions and programs, Decision Making in the Environmental Protection Agency, prepared for the Committee on Environmental Decision Making, National Academy of Sciences, Washington, D.C..

Richardson, Jeff, and John McKie, 2003, The rule of rescue, Social Science and Medicine 56, 2407-2419.

Samuelson, Paul A., 1954, The theory of pure provision of public expenditures, Review of Economics and Statistics 36, 387-389.

Schelling, Thomas, 1968, The life you save may be your own, Problems in Public Expenditure Analysis (S.B. Chase, Jr., ed.), Brookings Institution, Washington, D.C.

Small, Deborah A., and George Lowenstein, 2003, Helping $a$ victim or helping the victim: altruism and identifiability, Journal of Risk and Uncertainty 26, 5-16.

Sunstein, Cass R., 2005, The Laws of Fear: Beyond the Precautionary Principle, Cambridge University Press, Cambridge.

Tal, Alon, 1998, Beyond the rhetoric of premeditated murder: toward a rational and compassionate environmentalist perspective about the ethics of risk assessment, Ecosystem Health 4: 170-176.

Tietenberg, Tom, 1996, Environmental justice, Environmental and Natural Resource Economics, Chapter 20, Harper Collins College Publishers, New York.

Tversky, Amos, and Daniel Kahneman, 1974, Judgment under uncertainty: heuristics and biases, Science 185, 1124-1131. 
Ulph, Alistair, 1982, The role of ex ante and ex post decisions in the valuation of life, Journal of Public Economics 18, 265-76.

US Office of Management and Budget, 2003, Circular A-4, Regulatory Analysis, Washington, DC.

Viscusi, W. Kip, 1992, Strategic and ethical issues in the valuation of life, Fatal Tradeoffs: Public and Private Responsibilities for Risk, Chapter 2, Oxford University Press, Oxford .

Viscusi, W. Kip, 2000a, Misuses and proper uses of hedonic values of life in legal contexts, Journal of Forensic Economics 13, 111-25.

Viscusi, W. Kip, 2000b, Risk equity, Journal of Legal Studies 29, 843-71.

Viscusi, W. Kip, and Joseph E. Aldy, 2003, The value of a statistical life: a critical review of market estimates throughout the world, Journal of Risk and Uncertainty 27, 5-76.

Weinstein, Milton C., Donald S. Shepard, and Joseph S. Pliskin, 1980, The economic value of changing mortality probabilities: a decision-theoretic approach, Quarterly Journal of Economics 94, 373-96.

Williams, Alan, 1979, A note on trying to value a life, Journal of Public Economics 10, 257-58.

Zeckhauser, Richard J., 1975, Procedures for valuing lives, Public Policy 23, 419-464.

Zeckhauser, Richard J., and Donald S. Shepard, 1976, Where now for saving lives? Law and Contemporary Problems 40, 5-45. 


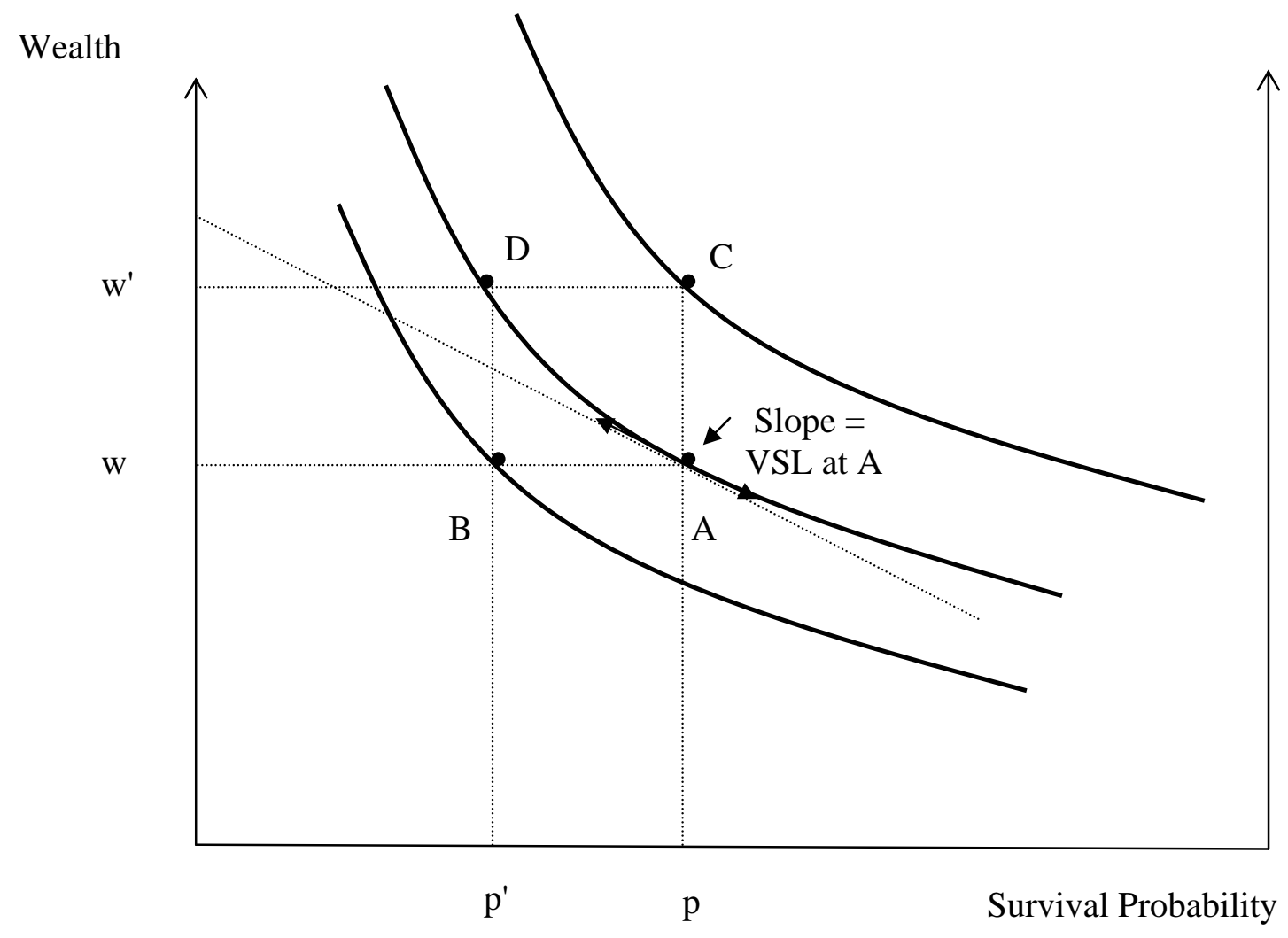

Figure 1: Indifference curves, VSL, the dead-anyway and wealth effects 